\title{
Convolutional Neural Network to Extract the Best Treatment Way of Warts Based on Data Mining
}

\author{
Muhammet Serdar Bascil \\ Department of Electrical and Electronics Engineering, Yozgat Bozok University, Yozgat 66200, Turkey
}

Corresponding Author Email: serdar.bascil@bozok.edu.tr

https://doi.org/10.18280/ria.330301

Received: 16 March 2019

Accepted: 2 June 2019

\section{Keywords:}

wart, cryotherapy, immunotherapy, convolutional neural network (CNN), data mining

\begin{abstract}
Cryotherapy and immunotherapy are two common methods to treat warts. This paper aims to determine which treatment methods is more effective in curing common types of warts. For this purpose, the author designed a convolutional neural network (CNN) to predict the effectiveness of wart treatment methods. The cryotherapy and immunotherapy datasets were randomly amplified by data mining rules, and represented as $2 \mathrm{D}$ grayscale pseudo images. The results show that the CNN with data mining can effectively predict the wart treatment effects; cryotherapy treatment is better than immunotherapy in curing common types of warts. This research is the first to combine $\mathrm{CNN}$ and data mining in predicting the treatment effects of warts.
\end{abstract}

\section{INTRODUCTION}

Warts are known as infectious disease of the skin caused by HPV [1]. They may grow on all part of the body and especially are rough, hard and skin color. Although the warts have structural differences such as appearance, color and size according to the region, they are generally categorized in four types as common, plantar, flat and genital $[2,3]$. The plantar and common warts are the most common types of them in the society and cryotherapy and immunotherapy methods are commonly used for the treatment of these species [4].

Cryotherapy is a simple and efficient method widely used for a long time and known as ice treatment. In this method, a probe point is cooled with the help of liquid nitrogen or carbon dioxide. Then, it is applied to compromise on the formation and provides the body to fight the virus. It may cause some adverse effects such as pain, color change on the skin and skin spots and it also take a long time on procedure of treatment [58]. Immunotherapy is a new method in comparison with cryotherapy. It is aimed to activate immune system of the patient to destroy warts with this method. For this purpose, an agent which the body shows allergic response is usually applied on the wart to wipe out it by activating the immune system [9-12].

It is crucial to predict convenient treatment method by utilizing disease and patient for physicians. For this purpose, computer based decision support systems have long been used in order to help medical experts to make decision and select more suitable treatment method. There are some studies of computer assisted precision trying to predict success of wart treatment method on plantar and common warts by using the same dataset created by cryotherapy and immunotherapy methods in literature. In the first study, Khozeimeh et al., created the dataset conducting on 180 patients. The 90 patients were treated by cryotherapy method and the others with immunotherapy method randomly. Then, they proposed a fuzzy logic rule-based system to predict treatment method and observed prediction accuracy on cryotherapy as $80.7 \%$ and immunotherapy as $83.33 \%$ [13]. On the next study of them,
T-test and chi-square test were used to extract statistical results. But, none of notable statistical difference were observed between groups. They finally reported that the immunotherapy method is more impressive than cryotherapy method based on it needs fewer sessions and capable of treating distant warts [14]. In the study of Akben, the decision tree-based algorithm was used to predict the success of wart treatment methods. He transformed decision tree rules into visual images as a function of patient age and the time elapsed since disease onset. The results of the study were compared with well-known classification methods and it was emphasized that the highest prediction results were obtained as $94.4 \%$ on cryotherapy dataset and $90 \%$ on immunotherapy dataset [15]. Tanyıldızı et al., were compared the performance of the classification algorithms on the same cryotherapy and immunotherapy dataset. They indicated that the K-Star algorithm is the best choice as the performance of $96.66 \%$ on the selection of cryotherapy treatment method and the Random Forest algorithm is the choice as the performance of $85.55 \%$ on the selection of immunotherapy treatment method [16].

In this work, Convolutional Neural Network (CNN) with data mining is used to predict the most convenient treatment way on plantar and common types of warts using cryotherapy and immunotherapy methods. The data mining rules were performed to randomly enlarge both of the dataset cryotherapy and immunotherapy. With the help of this method, the numerical data are represented as visual images. To the best of author knowledge, it is the first study on data mining combining with $\mathrm{CCN}$ to predict the treatment method success as percentage. The results of the study are also compared with previous studies which used same cryotherapy and immunotherapy dataset.

\section{MATERIALS}

The datasets used in the study created by Khozeimeh et al., in the dermatology clinic of Ghaem Hospital in Mashhad from January 2013 to February 2015 with plantar and common 
warts. The first dataset consists of six features and one result collected from 90 patients treated by cryotherapy method (with liquid nitrogen). The second one has seven features and one result collected from 90 patients treated by immunotherapy method (with candida antigen). The related datasets are given in Table 1, detailed. Both of the them are randomly collected from 180 patients consulted on dermatology clinic. Also, the datasets are available in the web site of UCI Machine Learning Repository as open-access under the headings of Cryotherapy Dataset [17] and Immunotherapy Dataset [18].

Table 1. The detailed cryotherapy and immunotherapy datasets

\begin{tabular}{|c|c|c|c|c|c|}
\hline \multicolumn{3}{|c|}{ Cryotherapy Dataset } & \multicolumn{3}{|c|}{ Immunotherapy Dataset } \\
\hline Features & Values & Mean \pm SD & Features & Values & Mean \pm SD \\
\hline $\begin{array}{l}\text { Gender (47 man, } 43 \\
\text { woman) }\end{array}$ & $\begin{array}{c}\operatorname{man} \text { is "1" } \\
\text { woman is "2" }\end{array}$ & & $\begin{array}{c}\text { Gender (41 man, } 49 \\
\text { woman) }\end{array}$ & $\begin{array}{c}\operatorname{man} \text { is "1" } \\
\text { woman is "2" }\end{array}$ & \\
\hline Age (year) & $15-67$ & $28.6 \pm 13.36$ & Age (year) & $15-56$ & $31.04 \pm 12.23$ \\
\hline $\begin{array}{l}\text { Time elapsed before } \\
\text { treatment (month) }\end{array}$ & $0-12$ & $7.66 \pm 3.4$ & $\begin{array}{l}\text { Time elapsed before } \\
\text { treatment (month) }\end{array}$ & $0-12$ & $7.23 \pm 3.10$ \\
\hline $\begin{array}{l}\text { The number of } \\
\text { warts }\end{array}$ & $1-12$ & $5.51 \pm 3.57$ & $\begin{array}{l}\text { The number of } \\
\text { warts }\end{array}$ & $1-19$ & $6.14 \pm 4.2$ \\
\hline $\begin{array}{l}\text { Types of wart } \\
\text { (Count) }\end{array}$ & $\begin{array}{c}\text { Common is "1" } \\
\text { Plantar is "2" } \\
\text { Both of them is "3" }\end{array}$ & & $\begin{array}{l}\text { Types of wart } \\
\text { (Count) }\end{array}$ & $\begin{array}{c}\text { Common is "1" } \\
\text { Plantar is "2" } \\
\text { Both of them is "3" }\end{array}$ & \\
\hline $\begin{array}{l}\text { Surface area of the } \\
\text { warts }\left(\mathrm{mm}^{2}\right)\end{array}$ & $4-750$ & $85.83 \pm 131.73$ & $\begin{array}{c}\text { Surface area of the } \\
\text { warts }\left(\mathrm{mm}^{2}\right)\end{array}$ & $6-900$ & $95.7 \pm 136.61$ \\
\hline & & & $\begin{array}{c}\text { Induration diameter } \\
\text { of initial test }(\mathrm{mm})\end{array}$ & $5-70$ & $14.33 \pm 17.22$ \\
\hline Result & \multicolumn{2}{|c|}{ Values } & Result & \multicolumn{2}{|c|}{ Values } \\
\hline $\begin{array}{l}\text { Response to } \\
\text { treatment }\end{array}$ & \multicolumn{2}{|c|}{$\begin{array}{l}\text { YES is equal " } 1 " \text { and } 48 \text { of them are "1" } \\
\text { NO is equal "0" and } 42 \text { of them are "0" }\end{array}$} & $\begin{array}{l}\text { Response to } \\
\text { treatment }\end{array}$ & \multicolumn{2}{|c|}{$\begin{array}{l}\text { YES is equal "1" and } 71 \text { of them are "1" } \\
\text { NO is equal "0" and } 19 \text { of them are "0" }\end{array}$} \\
\hline
\end{tabular}

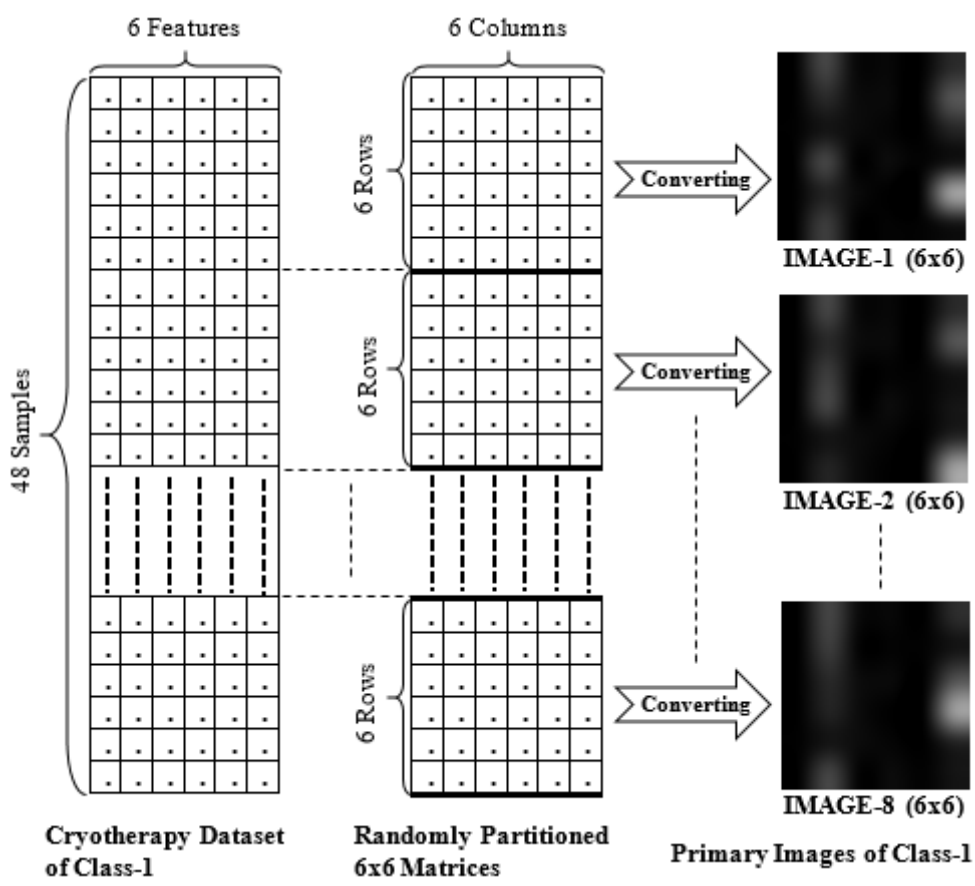

Figure 1. Primary images of cryotherapy dataset on class-1

\section{METHODS}

\subsection{Data mining}

The cryotherapy and immunotherapy datasets used in the study have two results Class- 1 and Class- 0 . Class- 1 is represent the success of treatment methods and Class- 0 is failure of them. Cryotherapy dataset consists of 48 samples of Class-1 and 42 samples of Class-0 with 6 features. Immunotherapy dataset composes of 71 samples of Class-1 and 19 samples of Class- 0 with 7 features. Figure 1 shows the primary image extractions of cryotherapy dataset on Class-1 as a sample to prevent confusion. The cryotherapy dataset of Class-1 (48x6) was randomly partitioned to small ones $(6 \times 6)$. Then, each of these small ones converted to create grayscale primary images of Class- 1 and 8 different primary images (6x6 image) were obtained seen on Figure 1. Similarly, 7 different primary images were formed on cryotherapy dataset of Class0 . The same way, 10 different primary images on immunotherapy dataset of Class- 1 and 2 different primary images on immunotherapy dataset of Class-0 were extracted. But, to be create similar structure of primary image on 
immunotherapy dataset (7x7 image), 1 and 5 residual samples were randomly discarded from Class-1 and Class-0, respectively.

Classifiers provides much more accurate results feeding with much more input data $[19,20]$. For this purpose, input dataset has to be enlarged using data mining techniques to supply and implement classifiers [21, 22]. In the study, pseudo images were derived from primary images shown in Figure 2 as a sample. Each of the $6 \times 6$ pseudo image is one of the permutations of primary images on cryotherapy dataset. All possible 720 different pseudo images were obtained from first primary image (IMAGE-1) of cryothera py dataset on Class-1. A total of 5760 (720x8) pseudo images are created on all cryotherapy dataset of Class-1 and 5040 (720x7) pseudo images on all cryotherapy dataset of Class-0. Also, each of the $7 \times 7$ pseudo image is one of the permutations of primary images on immunotherapy dataset. A total of 50400 (5040x 10) pseudo images are created on all immunotherapy dataset of Class-1 and $10080 \quad(5040 \times 2)$ pseudo images on all immunotherapy dataset of Class-0.

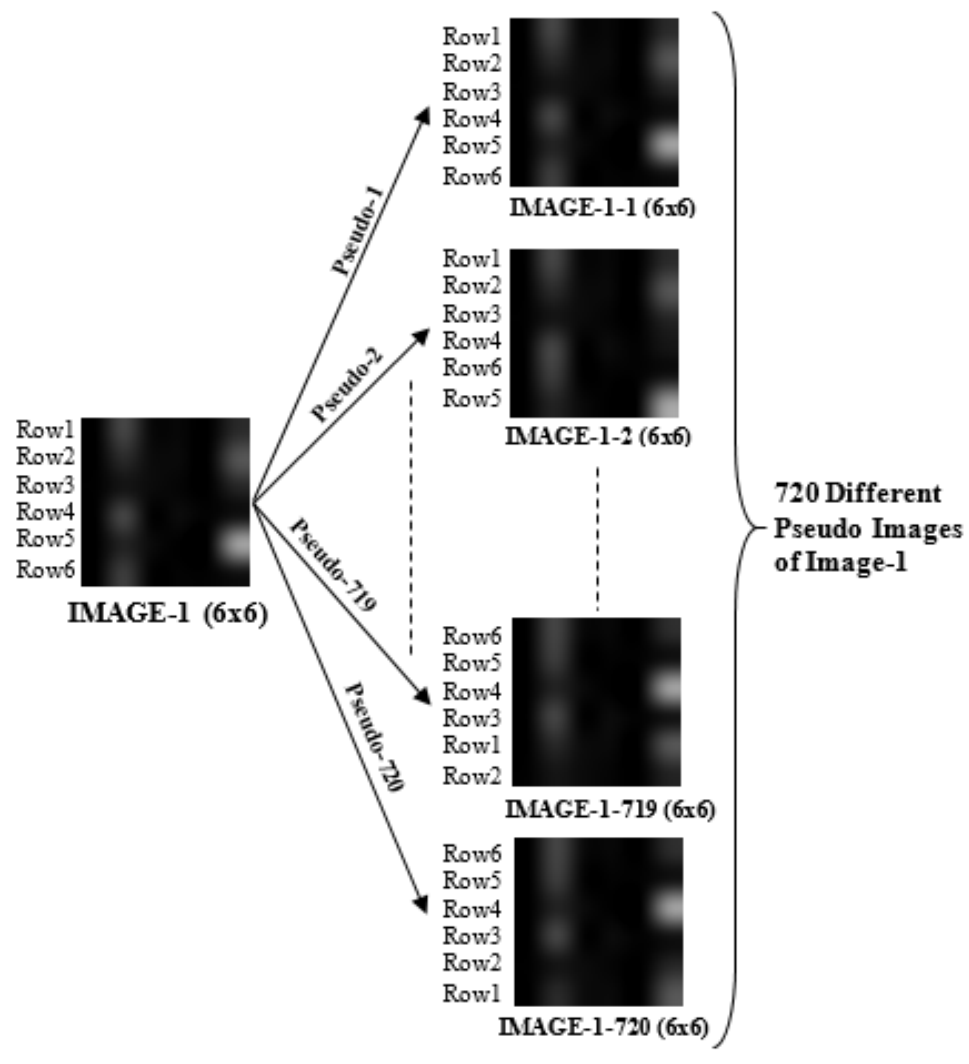

Figure 2. Pseudo images of the first primary image (image-1) on cryotherapy dataset of class-1

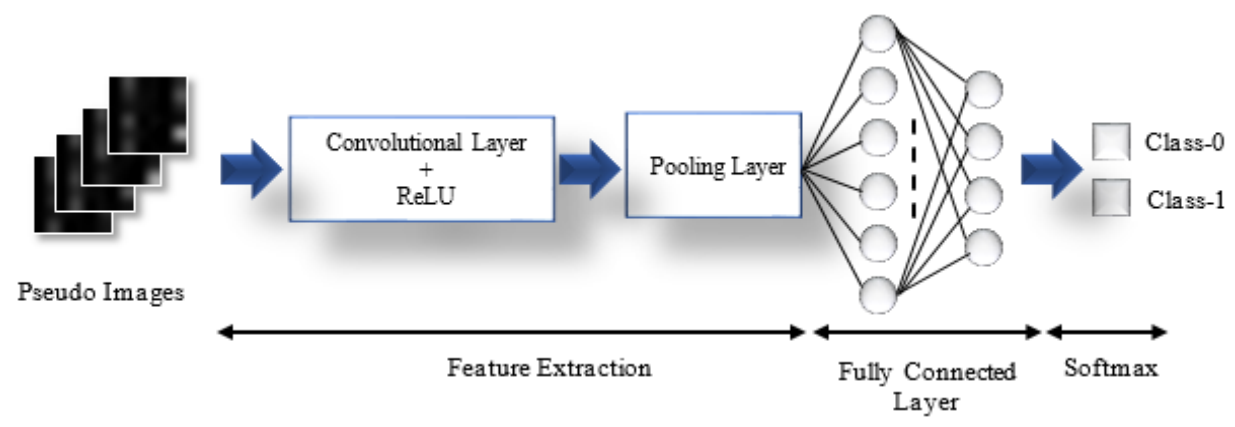

Figure 3. The concept of CNN model

\subsection{Convolutional Neural Network (CNN)}

Convolutional Neural Network (CNN) is a deep learning feed forward artificial neural network model. It has marked a new epoch in computer vision, classification and machine learning in recent years and it is forceful in pattern recognition of image. It uses unique features that make a subject to distinguish the image given such as on the insight of visual cortex of brain [23-25]. CNN performs the input data on three major layers that are convolutional layer, pooling layer, and a fully connected layer seen on Figure 3 . The convolutional layer is the basic layer of $\mathrm{CNN}$ responsible to extract unique features of image such as the edges, lines and corners. With this mind, CNN uses filters which are the matrix to be convolved with the pixels of image [16]. The pooling layer is known as down-sampling layer and used to reduce dimension of features. So, this layer minimizes computational complexity and overfitting [26, 27]. Then, the fully connected layer is performed to compute class scores.

Two types activation function gives the best results for speed of network training Rectifier (ReLu) and Softmax is used in the study. 
Rectified Linear Activation Unit (ReLU): There is common practice to apply an activation function after each convolutional layer. ReLU provides nonlinearity to the network structure given in Eq. 1 [25, 27].

$$
f(x)=\left\{\begin{array}{c}
x, \quad x>0 \\
0, \quad x<0
\end{array}\right.
$$

Softmax: This function is used to compute the probability of the $\mathrm{k}$ output class. The related equation of softmax is represented in Eq. 2.

$$
p_{j}=\frac{e^{x_{j}}}{\sum_{1}^{k} e^{x_{k}}} \quad j=1, \ldots k
$$

where, $x$ is the input of the net. The values of the output are in the range of 0 and 1 and their summation is 1 [25].

The CNN architecture used in the study has included one convolution layer with the kernel size as 5 in different number of kernel numbers from 1 to 30. Moreover, the maximum pooling layer kernel size was 3 and the stride number was chosen as 2 .

The classification performances of $\mathrm{CNN}$ were obtained with the given formula in (3) and (4).

$$
\begin{gathered}
\operatorname{Classif.\operatorname {accuracy}}(N)=\frac{\sum_{i=1}^{|N|} \operatorname{assess}\left(n_{i}\right)}{|N|}, \quad n_{i} \in N \\
\operatorname{assess}(n)= \begin{cases}1 & \text { if classify }(n)=n c \\
0 & \text { otherwise }\end{cases}
\end{gathered}
$$

where, $\mathrm{N}$ is the set of data items to be classified (the test set), $\mathrm{n} \in \mathrm{N}, \mathrm{nc}$ is the class of the item $\mathrm{n}$, and classify(n) returns the classification of $n$ by $\mathrm{CNN}$.

Also, the sensitivity (SENS) which is the proportion of correctly classified Class- 1 and specificity (SPEC) which is the proportion of correctly classified Class- 0 values were used to test the performance of proposed CNN model shown in the equations of 5 and 6 [28].

$$
\begin{aligned}
& \text { Sensitivity }=\frac{T P}{T P+F N} \\
& \text { Specificity }=\frac{T N}{T N+F P}
\end{aligned}
$$

True positive (TP) is known as the count of correctly classified Class-1, true negative (TN) is the count of correctly classified Class-0, false positive (FP) is the count of incorrectly classified Class-1, and false negative $(\mathrm{FN})$ is the count of incorrectly classified class-0 [28]. The traditional $50 \%$ training and $50 \%$ test set was selected.

\section{RESULTS AND DISCUSSION}

In this study, the proposed $\mathrm{CCN}$ with data mining method is validated on cryotherapy and immunotherapy datasets to predict most convenient treatment way on plantar and common types of warts. Cryotherapy dataset 48 samples of Class- 1 and 42 samples of Class- 0 with 6 features collected from 90 patients, randomly partitioned 6x6 pieces and grayscale primary image. Then, each one of these images enlarged subsamples and 5760 pseudo images on Class- 1 and 5040 pseudo images on Class- 0 were created. In the same way, immunotherapy dataset 71 samples of Class- 1 and 19 samples of Class- 0 with 7 features collected from 90 patients, randomly partitioned $7 \times 7$ pieces and each of the pieces converted grayscale primary image. At the end, 10080 different pseudo images on Class-0 and 50400 different pseudo images on Class-1 were extracted. Also, immunotherapy dataset on Class-0 with 19 samples were randomly split maximum 2 pieces of $7 \times 7$ primary image size and Class- 1 with 71 samples were randomly split maximum 10 pieces of $7 \times 7$ primary image size. For this reason, 5 residual samples were discarded from Class-0 and 1 from Class-1. Finally, the proposed CNN structure was executed to obtain performance results on both of the pseudo images of cryotherapy and immunotherapy datasets and the performance results were given in Table 2.

Table 2. The performance results of $\mathrm{CNN}$

\begin{tabular}{|c|c|c|}
\hline Methods (CNN) \% & Cryotherapy & Immunotherapy \\
\hline Accuracy & 99.38 & 97.67 \\
\hline Sensitivity & 100 & 99.56 \\
\hline Specificity & 98.89 & 97.46 \\
\hline
\end{tabular}

The performance result of cryotherapy dataset is clearly more (1.71\% classification accuracy) and better than immunotherapy dataset seen on Table 2, although the total pseudo images of immunotherapy (60480) are 5.6 times more than cryotherapy (10800). It can be said that the cryotherapy treatment method is more convenient on plantar and common types of warts thanks to the proposed structure. Also, the mandatorily discarded datasets of immunotherapy from Class0 (5 residual samples) and Class-1 (1 residual sample) maybe effect the results of immunotherapy performances.

Table 3. The comparison of classification accuracies on the same cryotherapy and immunotherapy datasets

\begin{tabular}{|c|c|c|c|}
\hline \multirow{2}{*}{ Study } & Method & \multicolumn{2}{|c|}{ Classification Accuracy (\%) } \\
\cline { 3 - 4 } & $\begin{array}{c}\text { Cryotherapy } \\
\text { Dataset }\end{array}$ & $\begin{array}{c}\text { Immunotherapy } \\
\text { Dataset }\end{array}$ \\
\hline$[13]$ & Fuzzy-logic & 80.7 & 83.33 \\
\hline$[14]$ & $\begin{array}{c}\text { T-test and chi- } \\
\text { square test }\end{array}$ & 56.7 & 76.6 \\
\hline$[16]$ & Decision tree & 94.4 & 90 \\
\hline$[16]$ & $\begin{array}{c}\text { K-Star and } \\
\text { Random forest }\end{array}$ & 96.6 & 85.55 \\
\hline $\begin{array}{c}\text { This } \\
\text { study }\end{array}$ & $\begin{array}{c}\text { CNN with data } \\
\text { mining }\end{array}$ & 99.38 & 97.67 \\
\hline \multicolumn{3}{|c|}{} \\
\hline
\end{tabular}

Furthermore, the number of primary images of immunotherapy dataset on Class- 0 may be increased by replacing randomly selected 2 different $7 \times 7$ matrices rows using 5 residual samples. But, the idea of primary image is lost this time. In addition, if immunotherapy dataset had been more balanced samples on Class- 0 and Class- 1 such as cryotherapy dataset, the results could be better. The proposed approach results were also compared with the other study results on the literature before given in Table 3 .

From Table 2 and 3, the classification accuracies were obtained with proposed CNN structure as $99.38 \%$ and $97.67 \%$ for cryotherapy and immunotherapy methods, respectively. The success rate of proposed structure is $2.78 \%$ higher than the nearest previous study for cryotherapy and $7.67 \%$ immunotherapy. It can be said that the proposed approach is superior than the others to predict the best treatment way on plantar and common types of warts using cryotherapy and immunotherapy treatment methods. Also, the 
immunotherapy classification accuracies of Ref. [13] and [14] are higher than cryotherapy but Ref. [15] and [16] are vice versa. According to this study results, cryotherapy treatment method may be the best choice.

\section{CONCLUSIONS}

This work aimed to predict the best treatment way on plantar and common types of warts using cryotherapy and immunotherapy treatment methods and improve the results of previous studies on the same goal. For his purpose, 2-D grayscale pseudo images of cryotherapy and immunotherapy datasets were created by data mining method and the classification results are obtained by proposed CNN structure. The results showed that this study on cryotherapy and immunotherapy datasets is the best of all the study on the literature before. It is clearly said that the $\mathrm{CCN}$ structure with data mining method is the best choice to predict wart treatment way. Also, the cryotherapy results are pretty higher than immunotherapy. Therefore, it would be better choice for physicians to utilize cryotherapy treatment method on plantar and common types of warts. In addition, the proposed approach is used in the literature first and it can be used as predecision support system for physicians on the other medical treatment methods.

\section{REFERENCES}

[1] Rowson, K.E., Mahy, B.W. (1967). Human papova (wart) virüs. Bacteriological Reviews, 31(2): 110-131.

[2] Cubie, H.A. (2013). Diseases associated with human papillomavirus infection. Virology, 445(1-2): 21-34. https://doi.org/10.1016/j.virol.2013.06.007

[3] Kardani, K., Bolhassani. A. (2018). Types of benign or malignant diseases associated with HPV infections. A. Bolhassani (Ed.), HPV Infections: Diagnosis, Prevention and Treatment. Bentham Science, Iran. https://doi.org/10.2174/97816810861701180101

[4] McGibbon, D. (2006). Rook's Textbook of Dermatology. 7th edition. Clinical and Experimental Dermatology, 31(1): $\quad 178-179 . \quad$ https://doi.org/10.1111/j.13652230.2005.02034.x

[5] Bourke, J.F., Berth-Jones, J., Hutchinson, P.E. (1995). Cryotherapy of common viral warts at intervals of 1,2 and 3 weeks. British Journal of Dermatology, 132(3): 433-436. https://doi.org/10.1111/j.13652133.1995.tb08678.x

[6] Larsen, P.Ø., Laurberg, G. (1996). Cryotherapy of viral warts. Journal of Dermatological Treatment, 7(1): 29-31. https://doi.org/10.3109/09546639609086866

[7] Connolly, M., Bazmi, K., O'connell, M., Lyons, J.F., Bourke, J.F. (2001). Cryotherapy of viral warts: a sustained 10 -s freeze is more effective than the traditional method. British Journal of Dermatology, 145(4): 554-557. https://doi.org/10.1046/j.13652133.2001.04449.x

[8] Ahmed, I., Agarwal, S., Ilchyshyn, A., Charles-Holmes, S., Berth-Jones, J. (2001). Liquid nitrogen cryotherapy of common warts: Cryo-spray vs. cotton wool bud. British Journal of Dermatology, 144(5): 1006-1009. https://doi.org/10.1046/j.1365-2133.2001.04190.x

[9] Phillips, R.C., Ruhl, T.S., Pfenninger, J.L., Garber, M.R.
(2000). Treatment of warts with Candida antigen injection. Archives of Dermatology, 136(10): 1274-1275. http://dx.doi.org/10.1001/archderm.136.10.1274-a

[10] Horn, T.D., Johnson, S.M., Helm, R.M., Roberson, P.K. (2005). Intralesional immunotherapy of warts with mumps, Candida, and trichophyton skin test antigens: A single-blinded, randomized, and controlled trial. Arch Dermatol, 141(5): 589-594. https://doi.org/10.1001/archderm.141.5.589

[11] Gamil, H., Elgharib, I., Nofal, A., Abd-Elaziz, T. (2010). Intralesional immunotherapy of plantar warts: Report of a new antigen combination. Journal of the American Academy of Dermatology, 63(1): 40-43. https://doi.org/10.1016/j.jaad.2009.07.023

[12] Majid, I., Imran S. (2013). Immunotherapy with intralesional Candida albicans antigen in resistant or recurrent warts: A study. Indian Journal of Dermatology, 58(5): 360-365. $\quad$ https://doi.org/10.4103/00195154.117301

[13] Khozeimeh, F., Alizadehsani, R., Roshanzamir, M., Khosravi, A., Layegh, P., Nahavandi, S. (2017). An expert system for selecting wart treatment method. Computers in Biology and Medicine, 81: 167-175. https://doi.org/10.1016/j.compbiomed.2017.01.001

[14] Khozeimeh, F., Azad, F.J., Oskouei, Y.M., Jafari, M., Tehranian, S., Alizadehsani, R., Layegh, P. (2017). Intralesional immunotherapy compared to cryotherapy in the treatment of warts. International Journal of Dermatology, 56(4): 474-478. https://doi.org/10.1111/ijd.13535

[15] Akben, S.B. (2018). Predicting the success of wart treatment methods using decision tree based fuzzy informative images. Biocybernetics and Biomedical Engineering, 38(4): 819-827. https://doi.org/10.1016/j.bbe.2018.06.007

[16] Tanyıldızı, E., Karabatak, M., Yıldırım, G., Özpolat, Z. (2018). Performance analysis of classification algorithms in wart treatment. Science and Engineering Journal of Firat University, 30(2): 249-256.

[17] UCI Machine Learning Repository - Cryotherapy Dataset.

https://archive.ics.uci.edu/ml/datasets/Cryotherapy+Dat aset $t_{2}$ accessed on Dec. 13, 2019.

[18] UCI Machine Learning Repository - Immunotherapy Dataset.

https://archive.ics.uci.edu/ml/datasets/Immunotherapy+ Dataset, accessed on Dec. 13, 2019.

[19] Enachescu, C., Miron, C.D. (2009). Handwritten digits recognition using neural computing. Scientific Bulletin of the Petru Maior University of Targu Mures, 6: 17.

[20] Sharma, N., Jain, V., Mishra, A. (2018). An analysis of convolutional neural networks for image classification. Procedia Computer Science, 132: 377-384. https://doi.org/10.1016/j.procs.2018.05.198

[21] Kaufmann, M. (2003). Data Mining. D. Pyle (Ed.), Business modelling and data mining. Elsevier Inc., Amsterdam, 273-530. https://doi.org/10.1016/B978155860653-1.50011-7

[22] Patel, S., Patel, H. (2016). Survey of data mining techniques used in healthcare domain. International Journal of Information, 6(1/2): 53-60. https://doi.org/10.5121/ijist.2016.6206

[23] Stutz, D. (2014). Understanding convolutional neural networks. InSeminar Report, Fakultät für Mathematik, 
Informatik und Naturwissenschaften Lehr-und Forschungsgebiet Informatik VIII Computer Vision.

[24] Wu, J. (2017). Introduction to convolutional neural networks. National Key Lab for Novel Software Technology Nanjing University, 5-23.

[25] Acharya, U.R., Oh, S.L., Hagiwara, Y., Tan, J.H., Adeli H. (2017). Deep convolutional neural network for the automated detection and diagnosis of seizure using EEG signals. Computers in Biology and Medicine, 100: 1-9. https://doi.org/10.1016/j.compbiomed.2017.09.017

[26] Yim, J., Ju, J., Jung, H., Kim, J. (2015). Image classification using convolutional neural networks with multi-stage feature. Advances in Intelligent Systems and
Computing, 345: 587-594. https://doi.org/10.1007/9783-319-16841-8_52

[27] Wen, L., Li, X., Gao, L., Zhang, Y. (2018). A new convolutional neural network-based data-driven fault diagnosis method. IEEE Transactions on Industrial Electronics, 65(7): 5990-5998. https://doi.org/10.1109/TIE.2017.2774777

[28] Gorur, K., Bozkurt, M.R., Bascil, M.S., Temurtas, F. (2018). Glossokinetic potential based tongue-machine interface for 1-D extraction using neural networks. Biocybernetics and Biomedical Eng., 38(3): 745-759. https://doi.org/10.1016/j.bbe.2018.06.004 